\title{
In Vivo Assessment of Brain White Matter Inflammation in Multiple Sclerosis with ${ }^{18}$ F-PBR111 PET
}

\author{
Alessandro Colasanti ${ }^{1,2}$, Qi Guo ${ }^{2,3}$, Nils Muhlert ${ }^{4,5}$, Paolo Giannetti ${ }^{1}$, Mayca Onega ${ }^{2}$, Rexford D. Newbould ${ }^{2}$, \\ Olga Ciccarelli ${ }^{4}$, Stuart Rison ${ }^{6}$, Charlotte Thomas ${ }^{6}$, Richard Nicholas ${ }^{1,6}$, Paolo A. Muraro ${ }^{1}$, Omar Malik ${ }^{6}$, \\ David R. Owen ${ }^{1}$, Paola Piccini ${ }^{1}$, Roger N. Gunn ${ }^{1,2}$, Eugenii A. Rabiner*2,3, and Paul M. Matthews ${ }^{* 1,7}$ \\ ${ }^{1}$ Division of Brain Sciences, Department of Medicine, Imperial College London, London, United Kingdom; ${ }^{2}$ Imanova Centre for \\ Imaging Sciences, London, United Kingdom; ${ }^{3}$ Centre for Neuroimaging Sciences, Institute of Psychiatry, King's College London, \\ London, United Kingdom; ${ }^{4}$ UCL Institute of Neurology, London, United Kingdom; ${ }^{5}$ School of Psychology and Cardiff University \\ Brain Research Imaging Centre, Cardiff University, Cardiff, United Kingdom; ' Imperial College Healthcare NHS Trust, London, \\ United Kingdom; and ${ }^{7}$ Neurosciences, GlaxoSmithKline, Brentford, United Kingdom
}

PET radioligand binding to the $18-\mathrm{kD}$ translocator protein (TSPO) in the brains of patients with multiple sclerosis (MS) primarily reflects activated microglia and macrophages. We previously developed genetic stratification for accurate quantitative estimation of TSPO using second-generation PET radioligands. In this study, we used ${ }^{18} \mathrm{~F}-\mathrm{PBR} 111 \mathrm{PET}$ and MR imaging to measure relative binding in the lesional, perilesional, and surrounding normal-appearing white matter of MS patients, as an index of the innate immune response. Methods: ${ }^{18} \mathrm{~F}-\mathrm{PBR} 111$ binding was quantified in $11 \mathrm{MS}$ patients and 11 age-matched healthy volunteers, stratified according to the rs6971 TSPO gene polymorphism. Fluid-attenuated inversion recovery and magnetization transfer ratio (MTR) MR imaging were used to segment the white matter in MS patients as lesions, perilesional volumes, nonlesional white matter with reduced MTR, and nonlesional white matter with normal MTR. Results: ${ }^{18}$ F-PBR 111 binding was higher in the white matter lesions and perilesional volumes of MS patients than in white matter of healthy controls $(P<0.05)$. Although there was substantial heterogeneity in binding between different lesions, a within-subject analysis showed higher ${ }^{18} \mathrm{~F}-\mathrm{PBR} 111$ binding in $\mathrm{MS}$ lesions $(P<0.05)$ and in perilesional $(P<0.05)$ and nonlesional white matter with reduced MTR $(P<$ $0.005)$ than in nonlesional white matter with a normal MTR. A positive correlation was observed between the mean ${ }^{18} \mathrm{~F}-\mathrm{PBR} 111$ volume of distribution increase in lesions relative to nonlesional white matter with a normal MTR and the MS severity score (Spearman $\rho=0.62, P<0.05)$. Conclusion: This study demonstrates that quantitative TSPO PET with a second-generation radioligand can be used to characterize innate immune responses in MS in vivo and provides further evidence supporting an association between the white matter TSPO PET signal in lesions and disease severity. Our approach is practical for extension to studies of the role of the innate immune response in MS for differentiation of antiinflammatory effects of new medicines and their longer term impact on clinical outcome.

Received Nov. 15, 2013; revision accepted Mar. 18, 2014.

For correspondence or reprints contact either of the following:

Alessandro Colasanti, Imanova Ltd., Hammersmith Hospital, Du Cane Rd., W12 0NN London, U.K.

E-mail: a.colasanti@imperial.ac.uk

Paul M Matthews, E515, Burlington Danes Bldg., Hammersmith Hospital,

Du Cane Rd., W12 ONN London, U.K.

E-mail: p.matthews@imperial.ac.uk

${ }^{*}$ Contributed equally to this work.

Published online Jun. 5, 2014.

COPYRIGHT (c) 2014 by the Society of Nuclear Medicine and Molecular Imaging, Inc.
Key Words: multiple sclerosis; TSPO; microglia; lesion; PET; MTR

J Nucl Med 2014; 55:1112-1118

DOI: 10.2967/jnumed.113.135129

A

prominent neuropathologic feature of multiple sclerosis (MS) is the activation of microglia, resident brain innate immune response cells, in the white matter (WM), particularly within demyelinating lesions but also extending into adjacent WM tissue (1-4).

MR imaging markers specifically associated with microglial and macrophage activation are limited. Correlational postmortem neuropathology and MR imaging in MS have confirmed that T1weighted gadolinium contrast enhancement and associated T2weighted hyperintensity changes in WM reflect the adaptive immune response but do not specifically inform on relative macrophage or microglial involvement (5). T2-weighted WM hyperintensities also are nonspecifically associated with other pathologic features including demyelination, axonal loss, and astrogliosis (6). A recent study found that subtle reductions in the MR imaging magnetization transfer ratio (MTR) in perilesional WM volumes are associated with increased microglia density (7). However, decreases in MTR reflect other changes in tissue microstructure, such as demyelination, as well and do not provide specific biomarker for innate immune activation.

PET radioligand binding to the $18-\mathrm{kD}$ translocator protein (TSPO) in the brains of patients with MS primarily reflects activated microglia and macrophages $(8,9)$. However, the interpretation of the findings of early studies, using the first-generation PET tracer ${ }^{11} \mathrm{C}-(R)$-PK11195, is limited by the rather poor signalto-noise ratio for this tracer and nonspecific binding, which challenge accurate quantitation of the signal $(8,10-12)$.

Several second-generation TSPO PET radioligands with improved signal-to-noise ratio relative to ${ }^{11} \mathrm{C}-(R)-\mathrm{PK} 11195$ have been developed (13). Unexpectedly, the first 2 studies using newer TSPO radioligands did not report increases in WM binding of MS patients relative to healthy controls $(14,15)$. However, the analyses did not take into consideration the population variation in binding of radioligands associated with the rs6971 TSPO gene single nucleotide polymorphism (SNP) (16). The heterogeneity of binding across carriers of different alleles may have contributed to the 
failure to differentiate brain TSPO radioligand binding between MS patients and healthy controls successfully.

${ }^{18}$ F-PBR111 is a second-generation TSPO ligand with promising imaging characteristics (17). A recent study showed increased ${ }^{18}$ F-PBR111 uptake corresponding specifically to activated microglia in experimental autoimmune encephalomyelitis (18). We have developed a robust approach for quantitative in vivo assessment of specific ${ }^{18}$ F-PBR111 binding to TSPO (19).

Here, we have used ${ }^{18}$ F-PBR111 PET coregistered with MR imaging to evaluate regional binding to WM in healthy volunteers and in MS patients who were stratified genetically according to the rs6971 SNP. On the basis of postmortem descriptions of activated microglia and macrophage distributions $(1,7,20)$, we predicted increased ${ }^{18} \mathrm{~F}-\mathrm{PBR} 111$ binding in regions of $\mathrm{T} 2$ hyperintense lesions, in the immediate perilesional volumes, and in nonlesional volumes with reduced MTR, compared with that in the WM of healthy volunteers and WM with a normal MTR (normal appearing) in MS patients. The reproducibility of ${ }^{18} \mathrm{~F}$-PBR111 signal also was assessed in 5 subjects (4 healthy volunteers and $1 \mathrm{MS}$ patient).

\section{MATERIALS AND METHODS}

\section{Study Design}

This was an open-label study in patients with relapsing-remitting MS and age-matched healthy volunteers ( $n=11$ per group; the supplemental data provide details [supplemental materials are available at http://jnm.snmjournals.org]). All subjects were genotyped for the rs6971 SNP, which determines variations in affinity between subjects (who express either a high affinity [HAB] or low affinity [LAB] or coexpression of both binding states $[\mathrm{MAB}]$ ) for second-generation TSPO radioligands (16). Patients with MS and healthy volunteers were matched for the rs6971 SNP (Supplemental Table 1).

Each participant underwent an MR imaging scan and an ${ }^{18} \mathrm{~F}-\mathrm{PBR} 111$ PET scan on the same day. Four healthy volunteers and 1 MS patient (all HABs) underwent a second ${ }^{18} \mathrm{~F}-\mathrm{PBR} 111$ PET scan on the following day to evaluate the reproducibility of the PET signal.

Disability was assessed by an experienced consultant neurologist using the expanded disability status scale. The MS severity score (MSSS) was calculated using expanded disability status scale scores and disease duration according to Roxburgh et al. (21).

\section{Imaging Methods}

Details on radioligand synthesis, PET protocol, and MR imaging protocol are described in the supplemental data.

${ }^{18} \mathrm{~F}-\mathrm{PBR} 111$ was injected as an intravenous bolus over approximately $20 \mathrm{~s}$ and PET emission data collected in 3-dimensional mode for $120 \mathrm{~min}$ after injection. The injected activity was similar in healthy volunteers and MS patients $(164.6 \mathrm{MBq} \pm 9$ and $169.7 \mathrm{MBq} \pm 12.4$ [mean $\pm \mathrm{SD}]$, respectively).

Definition of Volumes of Interest (VOIs). The definition of the whole WM and T2 fluid-attenuated inversion recovery (FLAIR) hyperintense WM volumes is described in detail in the supplemental methods.

The remaining WM in the MS patients (remaining after exclusion of the T2 FLAIR hyperintense lesions) was further segmented to 3 VOIs as follows: perilesional volumes, including voxels within a 6-mm-thick volume disposed around T2 hyperintense lesions (Figs. 1A and 1B); nonlesional low MTR (NLLM) voxels, with MTR ranging between $90 \%$ and $98 \%$ of the nonlesional mean WM MTR (7) (Fig. 1C); and nonlesional high MTR (NLHM) voxels, showing an MTR of $98 \%$ or greater of the nonlesional mean WM MTR (7). The NLHM VOI was assumed to be representative of the normal WM tissue.

PET Quantification. Dynamic PET data were corrected for motion via frame-to-frame image registration and aligned with the individual's

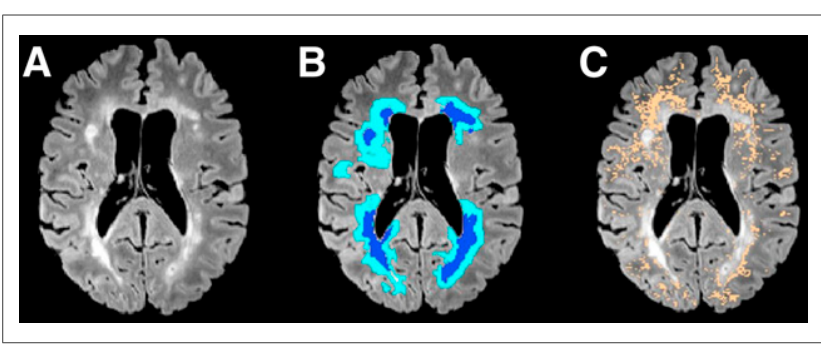

FIGURE 1. (A) T2 FLAIR image of representative MS patient (patient 1 , Supplemental Table 1). Hyperintense areas correspond to demyelinating lesions. (B) Lesion (dark blue) and perilesional areas (light blue) corresponding to intersection of 6-mm-diameter sphere traced around lesions within image plane. (C) Nonlesional voxels with MTR values ranging between $90 \%$ and $98 \%$ of mean MTR of nonlesional WM tissue (NLLM) (copper).

structural T1 MR image using SPM5 (Wellcome Trust Center for Neuroimaging, http://www.fil.ion.ucl.ac.uk/spm) with a mutual information cost function.

A 2-tissue-compartment kinetic model with a metabolite-corrected plasma input function was applied to the dynamic PET data using a fixed blood volume correction of 5\% (19). For each VOI examined, the total volume of distribution $\left(\mathrm{V}_{\mathrm{T}}\right)$ was estimated from the rate constants as described previously (22). The Logan graphical method (23) using a plasma input, with a fixed blood volume correction of 5\% and a linear start time at $35 \mathrm{~min}$, was also used to estimate the $\mathrm{V}_{\mathrm{T}}$ of each VOI and further applied at the voxel level to produce parametric $\mathrm{V}_{\mathrm{T}}$ maps. Model fitting and parameter estimation were performed using software implemented in Matlab R2008b (The MathWorks Inc.).

\section{Summary of Statistics}

We expressed ${ }^{18} \mathrm{~F}-\mathrm{PBR} 111 \mathrm{~V}_{\mathrm{T}}$ for the whole $\mathrm{WM}$ as $\mathrm{V}_{\mathrm{T}}^{\mathrm{WM}}$. For each subject, the mean ${ }^{18} \mathrm{~F}$-PBR111 $\mathrm{V}_{\mathrm{T}}$ uptake of all lesions larger than 100 voxels $\left(\mathrm{V}_{\mathrm{T}}^{\mathrm{L}}\right)$ and perilesional volumes $\left(\mathrm{V}_{\mathrm{T}}^{\mathrm{PL}}\right)$, respectively, was computed. The uptake of ${ }^{18} \mathrm{~F}$-PBR111 in NLLM and NLHM was expressed as $V_{T}^{N L L M}$ and $V_{T}^{N L H M}$, respectively. We used nonparametric statistic analysis with contrasts of $\mathrm{V}_{\mathrm{T}}^{\mathrm{WM}}$ in healthy controls to each of the MS patients' regions of interest $\left(\mathrm{V}_{\mathrm{T}}^{\mathrm{WM}}, \mathrm{V}_{\mathrm{T}}^{\mathrm{L}}, \mathrm{V}_{\mathrm{T}}^{\mathrm{PL}}, \mathrm{V}_{\mathrm{T}}^{\mathrm{NLLM}}\right.$, and $\mathrm{V}_{\mathrm{T}}^{\mathrm{NLHM}}$ ). Similarly we used nonparametric tests for the contrasts within MS patients $\left(\mathrm{V}_{\mathrm{T}}^{\mathrm{L}}, \mathrm{V}_{\mathrm{T}}^{\mathrm{PL}}\right.$, and $\mathrm{V}_{\mathrm{T}}^{\mathrm{NLL}}$ were separately compared with $\mathrm{V}_{\mathrm{T}}^{\mathrm{NLHM}}$ ). Details of the statistical analyses are presented in the supplemental methods. The relative increase $(\Delta)$ in ${ }^{18}$ F-PBR111 uptake in lesions, perilesional, and NLLM volumes versus NLHM was computed as follows:

$$
\begin{aligned}
\Delta_{\mathrm{L}} & =\left(\mathrm{V}_{\mathrm{T}}^{\mathrm{L}}-\mathrm{V}_{\mathrm{T}}^{\mathrm{NLHM}}\right) / \mathrm{V}_{\mathrm{T}}^{\mathrm{NLHM}} \\
\Delta_{\mathrm{PL}} & =\left(\mathrm{V}_{\mathrm{T}}^{\mathrm{PL}}-\mathrm{V}_{\mathrm{T}}^{\mathrm{NLHM}}\right) / \mathrm{V}_{\mathrm{T}}^{\mathrm{NLHM}} \\
\Delta_{\mathrm{NLLM}} & =\left(\mathrm{V}_{\mathrm{T}}^{\mathrm{NLLM}}-\mathrm{V}_{\mathrm{T}}^{\mathrm{NLHM}}\right) / \mathrm{V}_{\mathrm{T}}^{\mathrm{NLHM}}
\end{aligned}
$$

The variability across individual lesions, within each MS patient, was expressed using the coefficient of variation $\left(\mathrm{C}_{\mathrm{V}}\right)$ in $\mathrm{V}_{\mathrm{T}}^{\mathrm{L}}$ across individual lesions, as follows:

$$
\mathrm{C}_{\mathrm{V}}=\operatorname{standard} \operatorname{deviation}\left(\mathrm{V}_{\mathrm{T}}^{\mathrm{L}}\right) / \operatorname{mean}\left(\mathrm{V}_{\mathrm{T}}^{\mathrm{L}}\right)
$$

The test-retest variability of $\mathrm{V}_{\mathrm{T}}^{\mathrm{WM}}$ was studied in 4 healthy volunteers and $1 \mathrm{MS}$ patient and was expressed as the absolute difference between the 2 consecutive-days scans divided by the mean of the 2 scans. 
For the MS patient who was studied twice in consecutive days, we assessed test-retest variability of individual $\mathrm{V}_{\mathrm{T}}^{\mathrm{L}}$ and $\mathrm{V}_{\mathrm{T}}^{\mathrm{PL}}$. Additionally, we calculated the test-retest variability of individual normalized lesional and perilesional distribution volume ratio (DVR) by normalizing the corresponding $\mathrm{V}_{\mathrm{T}}$ values by $\mathrm{V}_{\mathrm{T}}^{\mathrm{NLHM}}$. The DVR was computed as follows:

$$
\begin{gathered}
\operatorname{DVR}_{\mathrm{L}}=\mathrm{V}_{\mathrm{T}}^{\mathrm{L}} / \mathrm{V}_{\mathrm{T}}^{\mathrm{NLHM}} \\
\mathrm{DVR}_{\mathrm{PL}}=\mathrm{V}_{\mathrm{T}}^{\mathrm{PL}} / \mathrm{V}_{\mathrm{T}}^{\mathrm{NLHM}}
\end{gathered}
$$

Details on correlational analyses are presented in the supplemental methods.

\section{RESULTS}

The demographic and clinical characteristics of study participants are presented in Supplemental Table 1. MS patients (10 women, 1 man) and healthy controls (5 women, 6 men) were matched for genotype ( $7 \mathrm{HABs}, 2 \mathrm{MABs}$, and $2 \mathrm{LABs}$ in each group) and had similar age distributions (healthy controls: median age, 51 y [range, 28-65 y] and MS patients: median age, $42 \mathrm{y}$ [range, 28-59 y]; Wilcoxon rank $P=0.80$ ).

\section{Between-Subjects Contrasts}

We observed a significant effect of TSPO genotype on WM binding of ${ }^{18} \mathrm{~F}-\mathrm{PBR} 111$ over the whole population $\left(\mathrm{V}_{\mathrm{T}}^{\mathrm{WM}}\right.$, HABs: median, 3.53 [range, 2.12-5.40], MABs: median, 3.00 [range, 2.504.43], and LABs: median, 1.47 [range, 1.18-1.70]; Kruskal-Wallis $P<0.01)$.

Whole WM. We saw a trend toward lower whole WM binding in healthy controls than in MS patients $\left(\mathrm{V}_{\mathrm{T}}^{\mathrm{WM}}\right.$, healthy controls: median, 2.50 [range, 1.18-5.23], MS patients: median, 3.53 [range, 1.62-4.55]; Wilcoxon rank $P=0.06$ ) (Fig. 2A; Supplemental Fig. 1). In MS patients, the $\mathrm{V}_{\mathrm{T}}^{\mathrm{WM}}$ correlated with disease duration in HABs (Fig. 2B) (Spearman $\rho=0.86 ; P=0.03$, corrected for age). The relationship between $\mathrm{V}_{\mathrm{T}}^{\mathrm{WM}}$ and disease duration was not assessed in the MAB and LAB groups separately, as each of these groups had only 2 subjects per group.

MR Imaging Segmentation-Based VOIs. One hundred sixtythree T2 FLAIR lesions were identified in the patients (median total lesion volume per patient, $12,708 \mathrm{~mm}^{3}$ [range, 392-32,672 $\left.\mathrm{mm}^{3}\right]$ ). Ninety-six of 163 individual lesions were larger than 100 voxels in volume (median number of lesions larger than 100 voxels per patient, $8 \mathrm{~mm}^{3}$; range, $1-17 \mathrm{~mm}^{3}$ ).

Both $\mathrm{V}_{\mathrm{T}}^{\mathrm{L}}$ (median, 3.88; range, 1.44-5.50) and $\mathrm{V}_{\mathrm{T}}^{\mathrm{PL}}$ (median, 3.61; range, 1.59-4.66) were greater than the $\mathrm{V}_{\mathrm{T}}^{\mathrm{WM}}$ from healthy controls (median, 2.50; range, 1.18-5.23; Wilcoxon rank $P=0.02$ and 0.03 , respectively, for $\mathrm{V}_{\mathrm{T}}^{\mathrm{L}}$ and $\mathrm{V}_{\mathrm{T}}^{\mathrm{PL}}$; Fig. 3). Direct contrasts between healthy controls' $\mathrm{V}_{\mathrm{T}}^{\mathrm{WM}}$ and MS patients' $\mathrm{V}_{\mathrm{T}}^{\mathrm{NLHM}}$ and $\mathrm{V}_{\mathrm{T}}^{\mathrm{NLLM}}$ demonstrated trends to greater binding in the patients' VOIs (Wilcoxon rank $P=0.09$ and 0.06, respectively) (Fig. 3; Supplemental Fig. 1).

\section{Within-Subjects Contrasts}

Relative ${ }^{18} \mathrm{~F}-\mathrm{PBR} 111 \mathrm{~V}_{\mathrm{T}}$ values for individual lesions and perilesional volumes, and in the NLHM and NLLM voxels, are presented separately for each MS patient (Fig. 4). The median $\mathrm{C}_{\mathrm{V}}$ in $\mathrm{V}_{\mathrm{T}}^{\mathrm{L}}$ across individual lesions within the patients was $15.2 \%$ (range, 9\%-25\%).

We found that $\mathrm{V}_{\mathrm{T}}$ in lesional $\left(\Delta_{\mathrm{L}}\right.$, median, $10.7 \%$ [range, -9.5 to 35.6]; Wilcoxon rank $P=0.03$ ), perilesional ( $\Delta_{\mathrm{PL}}$, median, $5.2 \%$ [range, -3.5 to 14.6 ]; Wilcoxon rank $P=0.01$ ), and NLLM vol-

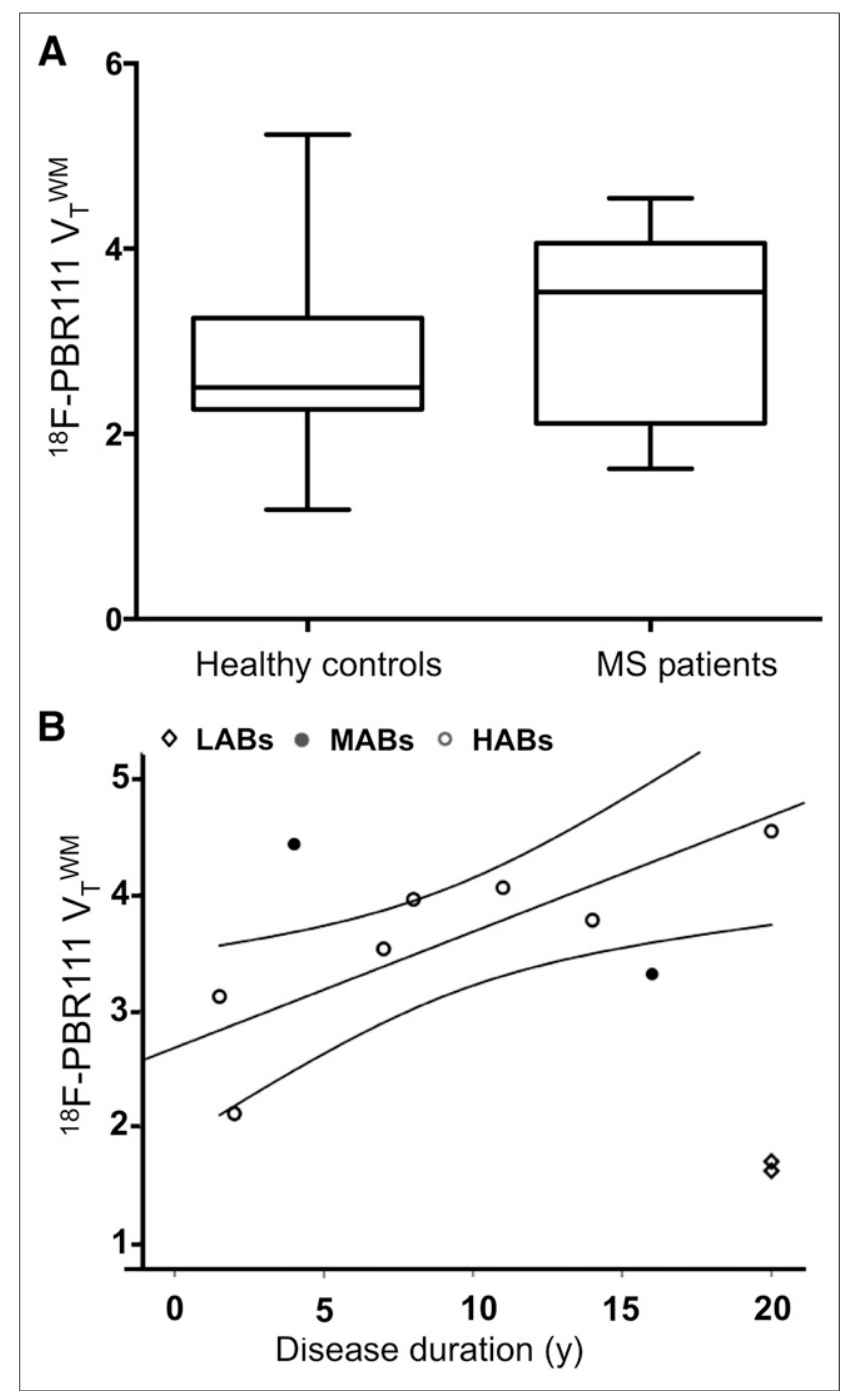

FIGURE 2. (A) ${ }^{18} \mathrm{~F}-\mathrm{PBR} 111 \mathrm{~V}_{\mathrm{T}}$ in whole $\mathrm{WM}$ of $\mathrm{MS}$ patients and genotype- and age-matched healthy control subjects. Lines in middle of boxes are median values, whereas hinges represent 25th and 75th percentiles, respectively. Whiskers represent maximum and minimum values. Contrast between MS patients and healthy controls showed trend for higher ${ }^{18} \mathrm{~F}-\mathrm{PBR} 111 \mathrm{~V}_{\mathrm{T}}^{\mathrm{WM}}$ in MS patients (Wilcoxon rank $P=$ 0.062). (B) Relationship between whole $W M{ }^{18} \mathrm{~F}-\mathrm{PBR} 111 \mathrm{~V}_{\mathrm{T}}$ and disease duration in MS patients for patients with different rs6971 genotypes (Spearman partial correlation in HABs: $\rho=0.86$; $P<0.05$, corrected for age).

umes $\left(\Delta_{\mathrm{NLLM}}\right.$, median, $1.2 \%$ [range, $0-2.4$ ]; Wilcoxon rank $P=$ $0.004)$ was increased relative to the $V_{T}$ in NLHM volumes within the MS patients. The maximum ${ }^{18} \mathrm{~F}-\mathrm{PBR} 111$ uptake increase relative to NLHM volumes was higher in lesions $(89 \%)$ than in the perilesional volumes (54\%). Sixty percent of lesions and $67 \%$ of perilesional volumes had higher ${ }^{18} \mathrm{~F}-\mathrm{PBR} 111 \mathrm{~V}_{\mathrm{T}}$ relative to NLHM.

Figures 5A-5C overlay locally thresholded ${ }^{18} \mathrm{~F}-\mathrm{PBR} 111 \mathrm{~V}_{\mathrm{T}}$ increases on T2 FLAIR hyperintense volumes in 2 patients with different clinical characteristics. In a patient who had experienced high disease activity (5 relapses in the last 2 y; Supplemental Table 1, patient 9), T2 FLAIR hyperintense lesion areas correspond to areas of increased ${ }^{18} \mathrm{~F}$-PBR111 signal. Conversely, in a patient with a more benign disease course and with no history of new neurologic symptoms reported over the past 2 y (Supplemental Table 1, 


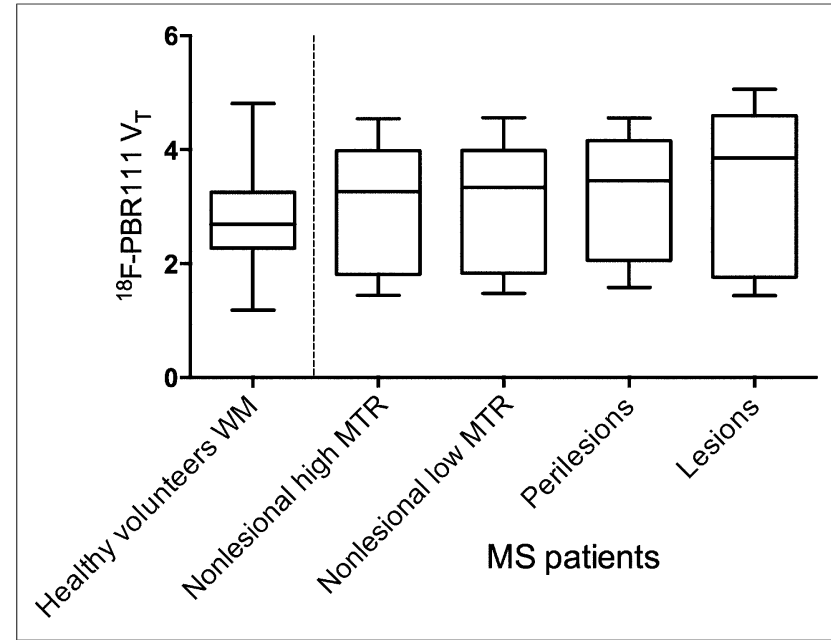

FIGURE 3. ${ }^{18} \mathrm{~F}-\mathrm{PBR} 111$ uptake in healthy volunteers and across MS patient ROIs. Lines in middle of boxes are median values, whereas hinges represent 25th and 75th percentiles, respectively. Whiskers represent maximum and minimum values. Between-group contrasts showed that MS patients' ${ }^{18} \mathrm{~F}-\mathrm{PBR} 111 \mathrm{~V} \mathrm{~V}$ and $\mathrm{V}_{\mathrm{T}}^{\mathrm{PL}}$ were greater than healthy volunteers' $\mathrm{V}_{T}^{\mathrm{WM}}$ (Wilcoxon rank $P<0.05$ ). Within-MS patient contrasts showed $V_{T}^{L}$ was greater than $\mathrm{V}_{T}^{\mathrm{NLHM}}$ and $\mathrm{V}_{T}^{\mathrm{PL}}$ was greater than $\mathrm{V}_{T}^{\mathrm{NLHM}}$ (Wilcoxon rank $P<0.05$ ), and $\mathrm{V}_{\mathrm{T}}^{\mathrm{NLLM}}$ was greater than $\mathrm{V}_{\mathrm{T}}^{\mathrm{NLHM}}$ (Wilcoxon rank $P<0.005$ ).

patient 4), focal areas of increased radiotracer binding correspond poorly with $\mathrm{T} 2$ hyperintense regions of WM (Figs. 5D-5F).

Disease severity, as assessed from MSSSs, correlated with the mean ${ }^{18} \mathrm{~F}-\mathrm{PBR} 111 \mathrm{~V}_{\mathrm{T}}$ increase in lesions for each patient (expressed relative to that in individual NLHM WM) (Spearman $\rho=0.62$; $P=0.05$; Fig. 6).

\section{Test-Retest Reproducibility}

The median test-retest variability in ${ }^{18} \mathrm{~F}-\mathrm{PBR} 111 \mathrm{~V}_{\mathrm{T}}^{\mathrm{WM}}$ across subjects (4 healthy volunteers, $1 \mathrm{MS}$ patient) was $23 \%$ (range, $12 \%-$ $55 \%$ ). The median test-retest variabilities in ${ }^{18} \mathrm{~F}-\mathrm{PBR} 111 \mathrm{~V}_{\mathrm{T}}^{\mathrm{L}}$ and $\mathrm{V}_{\mathrm{T}}^{\mathrm{PL}}$ across the $18 \mathrm{~T} 2$ hyperintense lesion volumes identified in the MS patient who was studied twice on consecutive days were $25 \%$ (range, $12 \%-57 \%$ ) and $27 \%$ (range, $17 \%-36 \%$ ), respectively, whereas the median test-retest variabilities for the normalized $\mathrm{DVR}^{\mathrm{L}}$ and $\mathrm{DVR}^{\mathrm{PL}}$ were $8 \%$ (range, $1 \%-31 \%$ ) and $4 \%$ (range, $0 \%-9 \%$ ), respectively.

\section{DISCUSSION}

TSPO PET can be used to assess the innate immune response in patients with MS in vivo, although methodologic and technical challenges have limited its wide application. We have illustrated how corrected, quantitative measures with a second-generation TSPO radioligand, ${ }^{18} \mathrm{~F}-\mathrm{PBR} 111$, promise new insights concerning clinicalpathologic correlations relevant to disease progression. We found increased ${ }^{18} \mathrm{~F}-\mathrm{PBR} 111 \mathrm{~V}_{\mathrm{T}}$ in the WM lesional and perilesional volumes of MS patients, compared with healthy volunteers. ${ }^{18} \mathrm{~F}-\mathrm{PBR} 111 \mathrm{~V}_{\mathrm{T}}$ was higher in lesions and perilesional and nonlesional volumes with decreased MTR (NLLM) in MS patients relative to the normal-appearing WM of the same subjects. Moreover, relative ${ }^{18} \mathrm{~F}-\mathrm{PBR} 111 \mathrm{~V}_{\mathrm{T}}$ increase in the MS lesions was positively correlated with disease severity, adding to recent in vivo data consistent with a role for the innate immune response in the progression of neurodegeneration in MS (24).

Postmortem autoradiographic studies in the brains of patients with MS have consistently demonstrated that increased uptake of

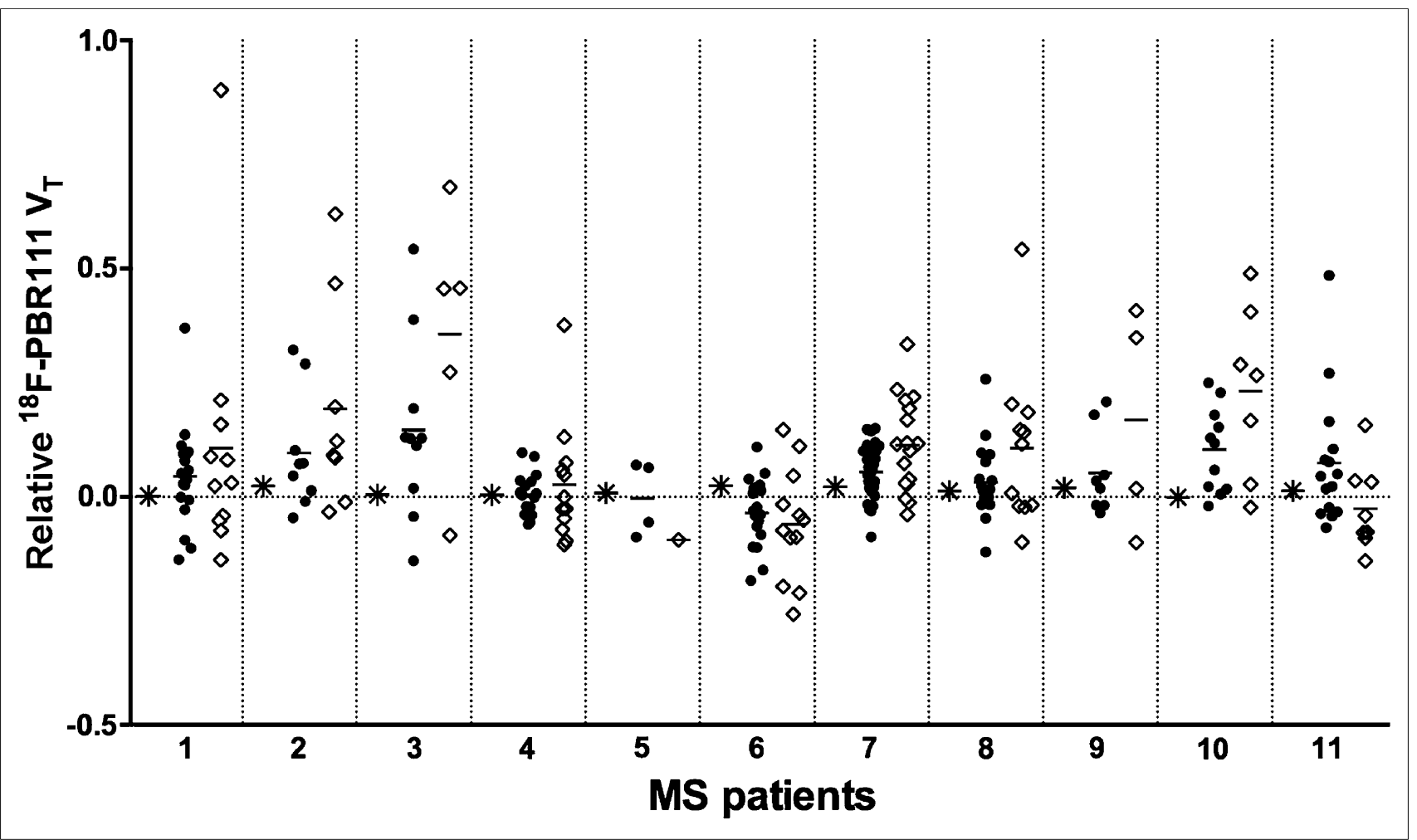

FIGURE 4. Relative ${ }^{18} \mathrm{~F}-\mathrm{PBR} 111$ uptake (relative to NLHM WM) in NLLM WM, in individual T2 FLAIR lesions, and in perilesional volumes for MS patients studied. Ordinant represents relative difference in ${ }^{18} \mathrm{~F}-\mathrm{PBR} 111 \mathrm{~V}_{\mathrm{T}}$ in lesions $(\diamond)$, in perilesional volumes $(\bullet)$, and in NLLM WM $\left({ }^{*}\right)$ relative to normal-appearing WM (NLHM WM). On abscissa, MS patients (Supplemental Table 1) are separately indicated. 


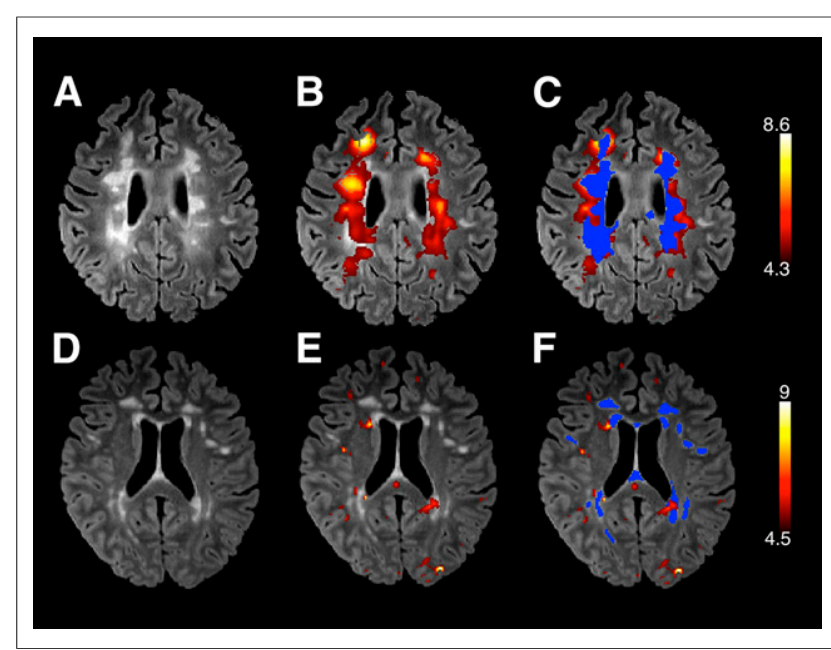

FIGURE 5. T2 FLAIR images ( $\mathrm{A}$ and $\mathrm{D}$ ), ${ }^{18} \mathrm{~F}-\mathrm{PBR} 111 \mathrm{~V}_{\mathrm{T}}$ parametric maps ( $B$ and $E$ ) overlaid (in warm colors) on T2 FLAIR images, and overlap between T2 FLAIR lesions (marked in blue) and ${ }^{18} \mathrm{~F}-\mathrm{PBR} 111$ $\mathrm{V}_{\mathrm{T}}$ (in warm colors) ( $\mathrm{C}$ and $\mathrm{F}$ ) from 2 illustrative patients. Lower threshold for $V_{T}$ parametric maps corresponds to value of ${ }^{18} \mathrm{~F}-\mathrm{PBR} 111 \mathrm{~V}_{\mathrm{T}}$ in NLHM volume for each of 2 patients. Upper threshold is twice $V_{T}$ in NLHM. Upper row illustrates patient with recent active disease (Supplemental Table 1, patient 9). Here, T2 FLAIR hyperintense lesional areas correspond to areas of increased ${ }^{18} \mathrm{~F}-\mathrm{PBR} 111$ signal. Lower row illustrates patient (Supplemental Table 1, patient 4) with relatively benign disease course showing focal regions of increased ${ }^{18} \mathrm{~F}-\mathrm{PBR} 111$ $\mathrm{V}_{\mathrm{T}}$ that correspond poorly to T2 FLAIR hyperintense areas.

TSPO-targeted radioligands colocalizes with markers of activated microglia $(8,11,25,26)$. An immunohistochemical analysis of postmortem MS brain tissue was largely consistent with these findings, suggesting that most cells expressing TSPO in acute MS lesions were macrophages or microglia (9), although it should be cautioned that antibody-based localization of the TSPO peptide and expression of the binding domain for the TSPO radioligands need not be the same. In a relapsing-remitting experimental autoimmune encephalomyelitis model (18), increased ${ }^{18} \mathrm{~F}-\mathrm{PBR} 111$ uptake colocalizes with activated microglia and macrophages and parallels the temporal dynamics of their upregulation during experimentally induced relapses.

We observed an increased ${ }^{18} \mathrm{~F}$-PBR111 uptake in approximately two thirds of MR imaging-defined lesions and perilesional volumes in MS patients relative to their normal-appearing WM (NLHM). Ex vivo pathology studies show that acute, active lesions are characterized by a hypercellular inflammatory core, marked by prominent lymphocyte infiltration with a high density of activated microglia and macrophages distributed evenly throughout the lesion (27). Patient 9 (Fig. 5, top) illustrates the strong colocalization of increased ${ }^{18} \mathrm{~F}-\mathrm{PBR} 111 \mathrm{~V}_{\mathrm{T}}$ with $\mathrm{T} 2$ hyperintense lesions in active disease. In chronic active lesions, microglia are increased relative to distant normal WM tissue and are more concentrated at the lesion edge than within the lesion. The hypercellular margin, characterized by a high density of activated microglia surrounding demyelinating plaques, is a consistent finding across neuropathologic studies $(2,28,29)$.

By contrast, approximately one third of the MR imagingdefined lesions and perilesional volumes were associated with ${ }^{18}$ F-PBR111 uptake similar to, or lower than, that of the normalappearing NLHM WM. Patient 4 (Fig. 5, bottom) is an illustrative example of poor correspondence between areas of increased ${ }^{18} \mathrm{~F}$ PBR111 $V_{T}$ and T2 FLAIR hyperintensities. We speculate that these volumes represent chronic inactive lesions that are hypocellular or have enlarged extracellular spaces leading to a relatively low density of all cells, including microglia. Our findings of regional variation thus are consistent with postmortem pathology observations in MS patients.

The median within-subject $\mathrm{C}_{\mathrm{V}}$ in lesional ${ }^{18} \mathrm{~F}-\mathrm{PBR} 111$ uptake was above $15 \%$. This finding indicates a moderately high variability of the observed ${ }^{18} \mathrm{~F}-\mathrm{PBR} 111$ signal between the T2 hyperintense lesions even within a single MS patient and further highlights that T2 hyperintense MR imaging contrast change reflects a wide range of neuropathology in MS lesions nonspecifically (30).

An elegant study by Moll et al. (7), using combined postmortem pathology and MR imaging, reported that regions appearing normal on T2-weighted MR, but displaying reduced MTR, were associated with microglial activation and axonal degeneration. By applying the same image segmentation approach as Moll et al., we observed a consistently increased ${ }^{18} \mathrm{~F}-\mathrm{PBR} 111$ uptake in regions with reduced MTR (NLLM) across our study group.

Focal areas of activated microglia identified neuropathologically in WM areas without apparent loss of myelin $(1,2)$ may represent areas at risk for the development of acute inflammatory lesions. They have been described previously as preactive lesions (5) or regions of chronic microglial activation that may contribute independently to progressive neurodegeneration (31). Alternatively, WM microglial activation in the absence of inflammatory demyelination may represent secondary reactive changes, perhaps associated with Wallerian degeneration (32). Direct tests of these alternative hypotheses now seem possible using serial MR imaging observations to follow the course of these WM changes identified by TSPO PET.

Although this work has gone further than previous studies in using a second-generation TSPO radioligand for MR image-correlated quantitative analyses, our findings are in general agreement with those of some TSPO-targeted PET studies in MS. Previous work using ${ }^{11} \mathrm{C}-(R)$-PK11195 demonstrated increased uptake to correspond to WM gadolinium-enhancing lesions $(8,10,11)$. One early

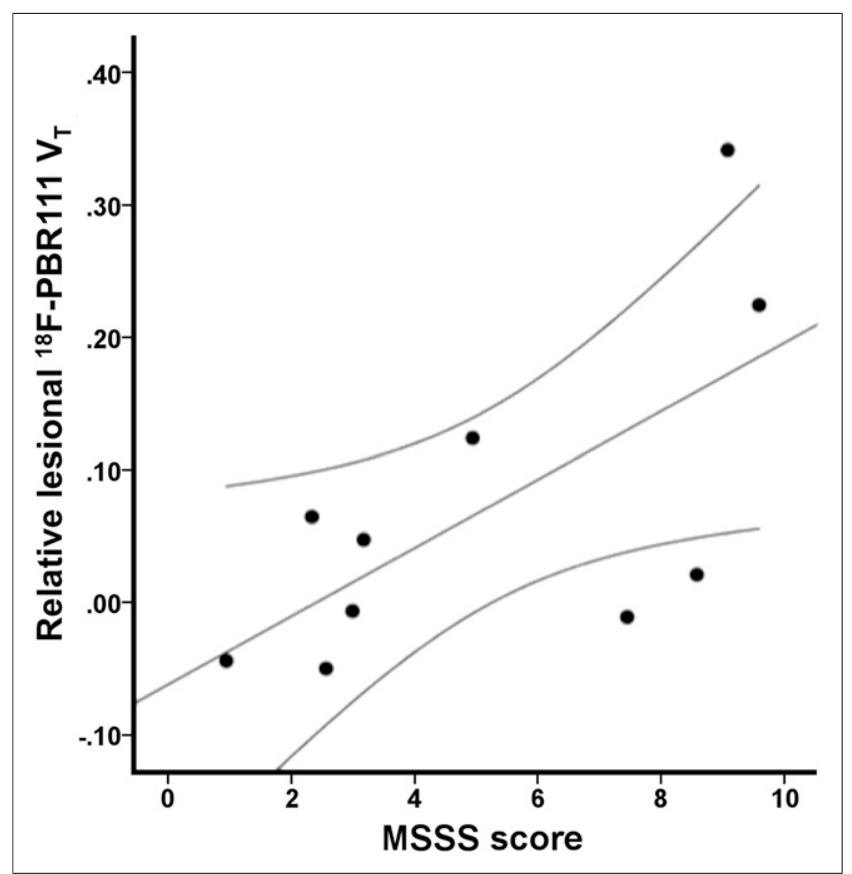

FIGURE 6. Positive relationship between MSSSs and ${ }^{18} \mathrm{~F}-\mathrm{PBR} 111$ uptake in lesions (expressed relative to that in normal-appearing WM) (Spearman $\rho=0.62, P \leq 0.05$ ). 
study suggested the presence of focal areas of increased uptake in normal-appearing white (and gray) matter (8). However, patterns of ligand uptake in T2-weighted hyperintensities and correlations between ${ }^{11} \mathrm{C}-(R)$-PK11195 uptake and disease duration and disability have been inconsistent in previous studies $(8,10,33)$. This inconsistency could reflect differences in patient populations, differences in the proportion of specific (displaceable) signal between ${ }^{18} \mathrm{~F}-\mathrm{PBR} 111$ and ${ }^{11} \mathrm{C}-(R)-\mathrm{PK} 11195$, or accurate modeling, which is particularly challenging for the lower-affinity ${ }^{11} \mathrm{C}-(R)$-PK11195 $(24,34)$.

This is the first study, to our knowledge, using a secondgeneration TSPO ligand to successfully detect significant differences in radioligand uptake between MS patients and healthy controls. The lack of differences seen in previous studies may be explained by failure to control for the variance introduced by the rs6971 TSPO SNP $(14,15)$.

There are several limitations of our study. We studied only a small number of patients. Further characterization of the heterogeneity of the disease is needed. The use of disease-modifying treatments in most MS patients studied may have influenced the ${ }^{18} \mathrm{~F}-\mathrm{PBR} 111$ signal. A study by Ratchford et al. showed a 3.2\% reduction in ${ }^{11} \mathrm{C}-(R)-\mathrm{PK} 11195$ in relapsing-remitting MS patients after $1 \mathrm{y}$ of treatment with glatiramer acetate, for example (35). This estimate of treatment effect is much lower than the differences in binding we found between healthy volunteers and patients overall, however ( $\sim 40 \%$ in the whole WM). However, in future work, it will be important to investigate the effects of various MS treatments on TSPO-specific binding.

MS patients and healthy controls were not matched for sex, although to our knowledge there have been no reports of a sex effect on TSPO binding in humans. Studies in rodents suggested a higher number of microglia and astrocytes in adult females $(36,37)$, so we cannot exclude the possibility that a higher prevalence of women in the MS group may have contributed to the higher ${ }^{18} \mathrm{~F}-\mathrm{PBR} 111 \mathrm{~V}_{\mathrm{T}}$ in patients.

Finally, increased TSPO is seen not just in activated microglia but also in rare, activated astrocytes (9) and in lymphocytes (38), as well as in brain vascular endothelia (39). The interpretation of increased ${ }^{18} \mathrm{~F}-\mathrm{PBR} 111 \mathrm{~V}_{\mathrm{T}}$ reported here as arising largely from activated macrophages or microglia is based on prior neuropathologic studies demonstrating large numbers of these cells postmortem in the regions studied and on the relatively high binding to them relative to other inflammatory cells $(2,27-29)$. A contribution from other inflammatory cell types cannot be excluded, but binding to lymphocytes is relatively much lower (40). Further technical aspects are considered in the supplemental data.

\section{CONCLUSION}

This study demonstrates that quantitative TSPO PET with a second-generation radioligand is sensitive to an element of the inflammatory response in MS not apparent on MR imaging and so otherwise occult in vivo. It highlights opportunities for the integration of MR imaging and molecular imaging for understanding the evolution of the dynamic neuropathology of MS.

\section{DISCLOSURE}

The costs of publication of this article were defrayed in part by the payment of page charges. Therefore, and solely to indicate this fact, this article is hereby marked "advertisement" in accordance with 18 USC section 1734. This study is supported by a GlaxoSmithKline/Wellcome Trust Fellowship in Translational Medicine and Therapeutics awarded through Imperial College London.
Paul M. Matthews is a part-time employee of GlaxoSmithKline, which contributed PET scanning time and operational support for the conduct of this study. No other potential conflict of interest relevant to this article was reported.

\section{ACKNOWLEDGMENTS}

We are extremely grateful to study participants for their patience and collaboration. We would also like to thank Awet Tewolde, Rahul Dimber, Yvonne Lewis, Michelle Cunneen, James Anscombe, Mark Tanner, and Ineke de Meer for their excellent technical support of this study. We also thank Bayer and ANSTO for providing preclinical data on ${ }^{18}$ F-PBR111 and the staff of Imanova and the Wellcome Trust NIHR McMichael Clinical Research Facility.

\section{REFERENCES}

1. Henderson AP, Barnett MH, Parratt JD, Prineas JW. Multiple sclerosis: distribution of inflammatory cells in newly forming lesions. Ann Neurol. 2009;66:739-753.

2. Marik C, Felts PA, Bauer J, Lassmann H, Smith KJ. Lesion genesis in a subset of patients with multiple sclerosis: a role for innate immunity? Brain. 2007;130:28002815.

3. van Horssen J, Singh S, van der Pol S, et al. Clusters of activated microglia in normal-appearing white matter show signs of innate immune activation. J Neuroinflammation. 2012;9:156.

4. Kutzelnigg A, Lucchinetti CF, Stadelmann C, et al. Cortical demyelination and diffuse white matter injury in multiple sclerosis. Brain. 2005;128:2705-2712.

5. De Groot CJ, Bergers E, Kamphorst W, et al. Post-mortem MRI-guided sampling of multiple sclerosis brain lesions: increased yield of active demyelinating and (p)reactive lesions. Brain. 2001;124:1635-1645.

6. Gouw AA, Seewann A, van der Flier WM, et al. Heterogeneity of small vessel disease: a systematic review of MRI and histopathology correlations. J Neurol Neurosurg Psychiatry. 2011;82:126-135.

7. Moll NM, Rietsch AM, Thomas S, et al. Multiple sclerosis normal-appearing white matter: pathology-imaging correlations. Ann Neurol. 2011;70:764-773.

8. Banati RB, Newcombe J, Gunn RN, et al. The peripheral benzodiazepine binding site in the brain in multiple sclerosis: quantitative in vivo imaging of microglia as a measure of disease activity. Brain. 2000;123:2321-2337.

9. Cosenza-Nashat M, Zhao ML, Suh HS, et al. Expression of the translocator protein of $18 \mathrm{kDa}$ by microglia, macrophages and astrocytes based on immunohistochemical localization in abnormal human brain. Neuropathol Appl Neurobiol. 2009;35:306-328.

10. Debruyne JC, Versijpt J, Van Laere KJ, et al. PET visualization of microglia in multiple sclerosis patients using [ [ ${ }^{11}$ C]PK11195. Eur J Neurol. 2003;10:257-264.

11. Vowinckel E, Reutens D, Becher B, et al. PK11195 binding to the peripheral benzodiazepine receptor as a marker of microglia activation in multiple sclerosis and experimental autoimmune encephalomyelitis. J Neurosci Res. 1997;50:345-353.

12. Vas A, Shchukin Y, Karrenbauer VD, et al. Functional neuroimaging in multiple sclerosis with radiolabelled glia markers: preliminary comparative PET studies with $\left[{ }^{11} \mathrm{C}\right]$ vinpocetine and $\left[{ }^{11} \mathrm{C}\right] \mathrm{PK} 11195$ in patients. J Neurol Sci. 2008;264:9-17.

13. Chauveau F, Boutin H, Van Camp N, Dolle F, Tavitian B. Nuclear imaging of neuroinflammation: a comprehensive review of $\left[{ }^{11} \mathrm{C}\right] \mathrm{PK} 11195$ challengers. Eur J Nucl Med Mol Imaging. 2008;35:2304-2319.

14. Oh U, Fujita M, Ikonomidou VN, et al. Translocator protein PET imaging for glial activation in multiple sclerosis. J Neuroimmune Pharmacol. 2011;6:354-361.

15. Takano A, Piehl F, Hillert J, et al. In vivo TSPO imaging in patients with multiple sclerosis: a brain PET study with [ ${ }^{18}$ F]FEDAA1106. EJNMMI Res. 2013;3:30.

16. Owen DR, Yeo AJ, Gunn RN, et al. An 18-kDa translocator protein (TSPO) polymorphism explains differences in binding affinity of the PET radioligand PBR28. J Cereb Blood Flow Metab. 2012;32:1-5.

17. Fookes CJ, Pham TQ, Mattner F, et al. Synthesis and biological evaluation of substituted $\left[{ }^{18} \mathrm{~F}\right]$ imidazo[1,2-a]pyridines and $\left[{ }^{18} \mathrm{~F}\right]$ pyrazolo $[1,5$-a $]$ pyrimidines for the study of the peripheral benzodiazepine receptor using positron emission tomography. J Med Chem. 2008;51:3700-3712.

18. Mattner F, Staykova M, Berghofer P, et al. Central nervous system expression and PET imaging of the translocator protein in relapsing-remitting experimental autoimmune encephalomyelitis. J Nucl Med. 2013;54:291-298.

19. Guo Q, Colasanti A, Owen DR, et al. Quantification of the specific translocator protein signal of $\left[{ }^{18} \mathrm{~F}\right] \mathrm{PBR} 111$ in healthy humans: a genetic polymorphism effect on in vivo binding. J Nucl Med. 2013;54:1915-1923.

20. Filippi M, Rocca MA, Barkhof F, et al. Association between pathological and MRI findings in multiple sclerosis. Lancet Neurol. 2012;11:349-360. 
21. Roxburgh RH, Seaman SR, Masterman T, et al. Multiple sclerosis severity score: using disability and disease duration to rate disease severity. Neurology. 2005;64:1144-1151.

22. Gunn RN, Gunn SR, Cunningham VJ. Positron emission tomography compartmental models. J Cereb Blood Flow Metab. 2001;21:635-652.

23. Logan J, Fowler JS, Volkow ND, et al. Graphical analysis of reversible radioligand binding from time-activity measurements applied to $\left[\mathrm{N}-{ }^{11} \mathrm{C}-\right.$ methyl]-(-)-cocaine PET studies in human subjects. J Cereb Blood Flow Metab. 1990;10:740-747.

24. Giannetti P, Politis M, Su P, et al. Microglia activation in multiple sclerosis black holes predicts outcome in progressive patients: an in vivo $\left[{ }^{11} \mathrm{C}\right](R)$-PK11195PET pilot study. Neurobiol Dis. 2014;65:203-210.

25. Venneti S, Wang G, Nguyen J, Wiley CA. The positron emission tomography ligand DAA1106 binds with high affinity to activated microglia in human neurological disorders. J Neuropathol Exp Neurol. 2008;67:1001-1010.

26. Owen DR, Howell OW, Tang SP, et al. Two binding sites for $\left[{ }^{3} \mathrm{H}\right] \mathrm{PBR} 28$ in human brain: implications for TSPO PET imaging of neuroinflammation. J Cereb Blood Flow Metab. 2010;30:1608-1618.

27. Trapp BD, Bo L, Mork S, Chang A. Pathogenesis of tissue injury in MS lesions. J Neuroimmunol. 1999;98:49-56.

28. Boyle EA, McGeer PL. Cellular immune response in multiple sclerosis plaques. Am J Pathol. 1990;137:575-584.

29. Lucchinetti C, Bruck W, Parisi J, Scheithauer B, Rodriguez M, Lassmann H. Heterogeneity of multiple sclerosis lesions: implications for the pathogenesis of demyelination. Ann Neurol. 2000;47:707-717.

30. Brück W, Stadelmann C. The spectrum of multiple sclerosis: new lessons from pathology. Curr Opin Neurol. 2005;18:221-224.
31. Smith KJ, Lassmann H. The role of nitric oxide in multiple sclerosis. Lancet Neurol. 2002;1:232-241.

32. Singh S, Metz I, Amor S, van der Valk P, Stadelmann C, Bruck W. Microglial nodules in early multiple sclerosis white matter are associated with degenerating axons. Acta Neuropathol. 2013;125:595-608.

33. Versijpt J, Debruyne JC, Van Laere KJ, et al. Microglial imaging with positron emission tomography and atrophy measurements with magnetic resonance imaging in multiple sclerosis: a correlative study. Mult Scler. 2005;11:127-134.

34. Yaqub M, van Berckel BN, Schuitemaker A, et al. Optimization of supervised cluster analysis for extracting reference tissue input curves in $(R)-\left[{ }^{11} \mathrm{C}\right] \mathrm{PK} 11195$ brain PET studies. J Cereb Blood Flow Metab. 2012;32:1600-1608.

35. Ratchford JN, Endres CJ, Hammoud DA, et al. Decreased microglial activation in MS patients treated with glatiramer acetate. J Neurol. 2012;259:1199-1205.

36. Mouton PR, Long JM, Lei DL, et al. Age and gender effects on microglia and astrocyte numbers in brains of mice. Brain Res. 2002;956:30-35.

37. Schwarz JM, Sholar PW, Bilbo SD. Sex differences in microglial colonization of the developing rat brain. J Neurochem. 2012;120:948-963.

38. Berkovich A, Ferrarese C, Cavaletti G, et al. Topology of two DBI receptors in human lymphocytes. Life Sci. 1993;52:1265-1277.

39. Turkheimer FE, Edison P, Pavese N, et al. Reference and target region modeling of $\left[{ }^{11} \mathrm{C}\right]-(R)-\mathrm{PK} 11195$ brain studies. J Nucl Med. 2007;48:158-167.

40. Canat X, Carayon P, Bouaboula M, et al. Distribution profile and properties of peripheral-type benzodiazepine receptors on human hemopoietic cells. Life Sci. 1993;52:107-118. 